\title{
Status of the MILC calculation of electromagnetic contributions to pseudoscalar masses
}

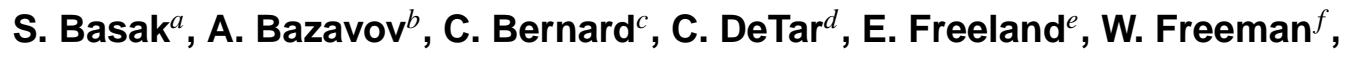 \\ J. Foley ${ }^{d}$, Steven Gottlieb ${ }^{g}$, U.M. Heller ${ }^{h}$, J.E. Hetrick ${ }^{i}$, J. Laiho ${ }^{j}$, L. Levkova ${ }^{* d}$, \\ M. Oktay ${ }^{d}$, J. Osborn ${ }^{k}$, R.L. Sugar ${ }^{l}$, A. Torok ${ }^{g}$, D. Toussaint ${ }^{m}$, R.S. Van de Water ${ }^{b}$, \\ R. Zhou ${ }^{g}$ (MILC Collaboration)
}

a NISER, Bhubaneswar, Orissa 751005, India

${ }^{b}$ Physics Department, Brookhaven National Laboratory, Upton, NY 11973, USA

c Physics Department, Washington University, St. Louis, MO 63130, USA

${ }^{d}$ Physics Department, University of Utah, Salt Lake City, UT 84112, USA

${ }^{e}$ Department of Physics, Benedictine University, Lisle, IL 60532, USA

${ }^{f}$ Department of Physics, George Washington University, Washington, DC 20037, USA

${ }^{g}$ Department of Physics, Indiana University, Bloomington, IN 47405, USA

${ }^{h}$ American Physical Society, One Research Road, Ridge, NY 11961, USA

${ }^{i}$ Physics Department, University of the Pacific, Stockton, CA 95211, USA

${ }^{j}$ SUPA, School of Physics and Astronomy, University of Glasgow, Glasgow, UK

${ }^{k}$ ALCF, Argonne National Laboratory, Argonne, IL 60439, USA

${ }^{l}$ Physics Department, University of California, Santa Barbara, CA 93106, USA

${ }^{m}$ Physics Department, University of Arizona Tucson, AZ 85721, USA

E-mail: Iudmila@physics.utah.edu

We calculate pseudoscalar masses on gauge configurations containing the effects of $2+1$ flavors of dynamical asqtad quarks and quenched electromagnetism. The lattice spacings vary from 0.12 to $0.06 \mathrm{fm}$. The masses are fit with staggered chiral perturbation theory including NLO electromagnetic terms. We attempt to extract the fit parameters for the electromagnetic contributions, while taking into account the finite volume effects, and extrapolate them to the physical limit.

The XXX International Symposium on Lattice Field Theory

July 24-29, 2012

Cairns, Australia

\footnotetext{
* Speaker.
} 


\section{Introduction}

Quark masses are fundamental parameters in the Standard Model and their determination from lattice field theory is important for phenomenology. With the advance of high-precision spectroscopy the electromagnetic (EM) contributions to the quark masses have become important. In fact, they constitute the largest uncertainty in the calculation of $m_{u} / m_{d}$ [1] as indicated in Table 1]. The EM error stems from uncertainties in the size of the EM contributions to the masses of the $\pi$ and especially the $K$ mesons. The contributions have until recently been taken from a variety of phenomenological estimates, and therefore have quite large and not well controlled errors. The MILC collaboration has been working on reducing these uncertainties and progress has been reported previously in Refs. [2, 3, 丹].

To lowest order (LO) in chiral perturbation theory $(\chi P T)$ the electromagnetic splitting in the pion and kaon systems is identical (this observation is known as Dashen's theorem [5]). We aim to calculate on the lattice the corrections to Dashen's theorem by employing staggered $\chi P T$ with EM effects for fits to spectrum data. The corrections to Dashen's theorem may be parametrized as [6]:

$$
\left(M_{K^{ \pm}}^{2}-M_{K^{0}}^{2}\right)^{\gamma}=(1+\varepsilon)\left(M_{\pi^{ \pm}}^{2}-M_{\pi^{0}}^{2}\right)^{\gamma}
$$

where the superscript $\gamma$ denotes the EM contributions. ${ }^{1}$ Our main motivation for computing $\varepsilon$ is to be able to remove the EM effects from the experimental $K$ masses; the resulting pure-QCD masses may be compared with QCD simulations in order to extract $m_{u} / m_{d}$. Staggered $\chi P T$, developed in Ref. [3], gives for the EM splitting between Goldstone pseudoscalars with charged and neutral quarks, $\Delta M_{x y, 5}^{2}$, the following NLO expression:

$$
\begin{aligned}
\Delta M_{x y, 5}^{2}= & q_{x y}^{2} \delta_{E M}-\frac{1}{16 \pi^{2}} e^{2} q_{x y}^{2} M_{x y, 5}^{2}\left[3 \ln \left(M_{x y, 5}^{2} / \Lambda_{\chi}^{2}\right)-4\right] \\
& -\frac{2 \delta_{E M}}{16 \pi^{2} f^{2}} \frac{1}{16} \sum_{\sigma, \xi}\left[q_{x \sigma} q_{x y} l\left(M_{x \sigma, \xi}^{2}\right)-q_{y \sigma} q_{x y} l\left(M_{y \sigma, \xi}^{2}\right)\right]+(\text { analytic terms })
\end{aligned}
$$

where $\sigma$ is the sea-quark label, $\xi$ is the staggered taste, $q_{x y}$ is the charge of a meson made of a quark $x$ and an antiquark $y$ (for more details on the notations see Ref. [3]). To fit our spectrum data,

\begin{tabular}{lccc}
\hline \hline & $m_{u}[\mathrm{MeV}]$ & $m_{d}[\mathrm{MeV}]$ & $m_{u} / m_{d}$ \\
\hline \hline value & 1.9 & 4.6 & 0.42 \\
statistics & 0.0 & 0.0 & 0.00 \\
lattice systematics & 0.1 & 0.2 & 0.01 \\
perturbative & 0.1 & 0.2 & - \\
EM & 0.1 & 0.1 & 0.04 \\
\hline \hline
\end{tabular}

Table 1: Value of $m_{u} / m_{d}$ calculated by the MILC Collaboration [1] and estimates of the different sources of error (with $m_{u}$ and $m_{d}$ determined in the $\overline{M S}$ scheme at $2 \mathrm{GeV}$ ). The row labeled "EM" shows the size of the error coming from a phenomenological estimate of electromagnetic contributions to the quark masses.

\footnotetext{
${ }^{1}$ We do not include quark-mass renormalization effects due to the presence of the EM field, since they are much smaller than our precision.
} 


\begin{tabular}{ccclr}
\hline \hline$\approx a[\mathrm{fm}]$ & Volume & $\beta$ & $m_{l} / m_{s}$ & \# configs. \\
\hline \hline 0.12 & $20^{3} \times 64$ & 6.76 & $0.01 / 0.05$ & 2254 \\
& $28^{3} \times 64$ & 6.76 & $0.01 / 0.05$ & 274 \\
& $20^{3} \times 64$ & 6.76 & $0.007 / 0.05$ & 1261 \\
& $24^{3} \times 64$ & 6.76 & $0.005 / 0.05$ & 2099 \\
\hline \hline 0.09 & $28^{3} \times 96$ & 7.09 & $0.0062 / 0.031$ & 1930 \\
& $40^{3} \times 96$ & 7.08 & $0.0031 / 0.031$ & 1015 \\
\hline \hline 0.06 & $48^{3} \times 144$ & 7.47 & $0.0036 / 0.018$ & 670 \\
\hline \hline
\end{tabular}

Table 2: Parameters of the asqtad ensembles used in this study.

we use the above expression plus the NNLO analytic terms. For investigating the finite volume effects, we include standard terms dependent on $m_{\pi} L_{s}$ from EM tadpoles, as well as an empirical EM finite volume correction in the form $f_{v} q_{x y}^{2} / L_{s}^{2}$ used previously in Ref. [7], where $f_{v}$ is a constant and $L_{s}$ is the spatial lattice size. However, as discussed below, our measured finite volume effects are rather small at present, and including or omitting the finite volume terms from the fits currently makes little difference in the final results.

\section{Lattice setup}

Our EM field on the lattice is "photon-quenched," i.e., the sea quarks are taken to be electromagnetically neutral, and the $U(1)_{\mathrm{EM}}$ links are generated independently of the $S U(3)_{\mathrm{QCD}}$ links. The fact that the photons are not present in the sea does not affect the NLO fits to the EM meson splittings since, as shown in Ref. [8], the unknown LEC controlling sea-quark mass dependence cancels in EM splittings between mesons with the same valence masses. There are physical NLO effects missing due to the absence of sea-quark charges, but these may be inserted after the fact since they appear only in chiral logarithm terms with known (LO) coefficients. Uncontrolled EM quenching effects do come in at NNLO, but should be negligible at the current level of precision. Because diagrams that have intermediate gluon or photon states ("quark-disconnected" diagrams) are difficult to compute on the lattice, and because of the photon quenching, we have to redefine the neutral pion on the lattice ' $\pi^{0 \prime}$ to have the mass:

$$
m_{\prime^{\prime \prime}}^{2}=\left(m_{u \bar{u}}^{2}+m_{d \bar{d}}^{2}\right) / 2,
$$

where $u \bar{u}$ and $d \bar{d}$ are states with charges $(2 / 3 e,-2 / 3 e)$ and $(1 / 3 e,-1 / 3 e)$ and disconnected diagrams are dropped by fiat. The effect of the disconnected diagrams is expected to be small, since EM contributions to the neutral pion must in any case vanish in the chiral limit.

We calculate the meson spectrum on the set of asqtad ensembles with $2+1$ flavors listed in Table 2 [1]. The values of the electron charge that we use are $e= \pm 0.909, \pm 0.606$ and, $\pm 0.303,0$, corresponding to $9 \alpha_{\text {phys }}, 4 \alpha_{\text {phys }}, \alpha_{\text {phys }}$ and 0 .

\section{Results}

We do not see large finite volume effects in the EM splittings calculated on the $a \approx 0.12 \mathrm{fm}$ 
ensembles with $L_{s}=20$ and 28 . The volume effects do increase with the quark charges, but remain small. In Fig. 1 we compare our results with the finite volume effects for a charged kaon (with 3 times the physical charges) expected from the BMW collaboration result [7 (magenta error bar). We find that ours are about three times smaller. A similar comparison is shown in Fig. 3 for physically charged kaons. Nevertheless, the error on the finite volume effect in our data (computed simply by propagating the errors in the difference between the results on the two volumes) is slightly larger than the effect itself, so any tension with the BMW result is less than a $2 \sigma$ effect. Indeed, we see no reason for any difference with BMW other than statistics, since as far as we can tell, we are treating the $U(1)_{\mathrm{EM}}$ fields in the same way they do, and we do not expect the EM finite volume effects to be strongly dependent on quark mass or quark action. The finite volume effects we see are also smaller than those expected from the RBC Collaboration results [9], but in that case some or all of the difference may be due to the different treatment of zero modes in our $U(1)_{\mathrm{EM}}$ fields. We are currently increasing the statistics on the $a \approx 0.12 \mathrm{fm}, L_{s}=28$ ensemble in hopes of clarifying this issue.

The taste splitting between the Goldstone pion and the second local pion grows with the quark charge for the neutral pions (see Fig. 国 for the two coarse ensembles with the same parameters and

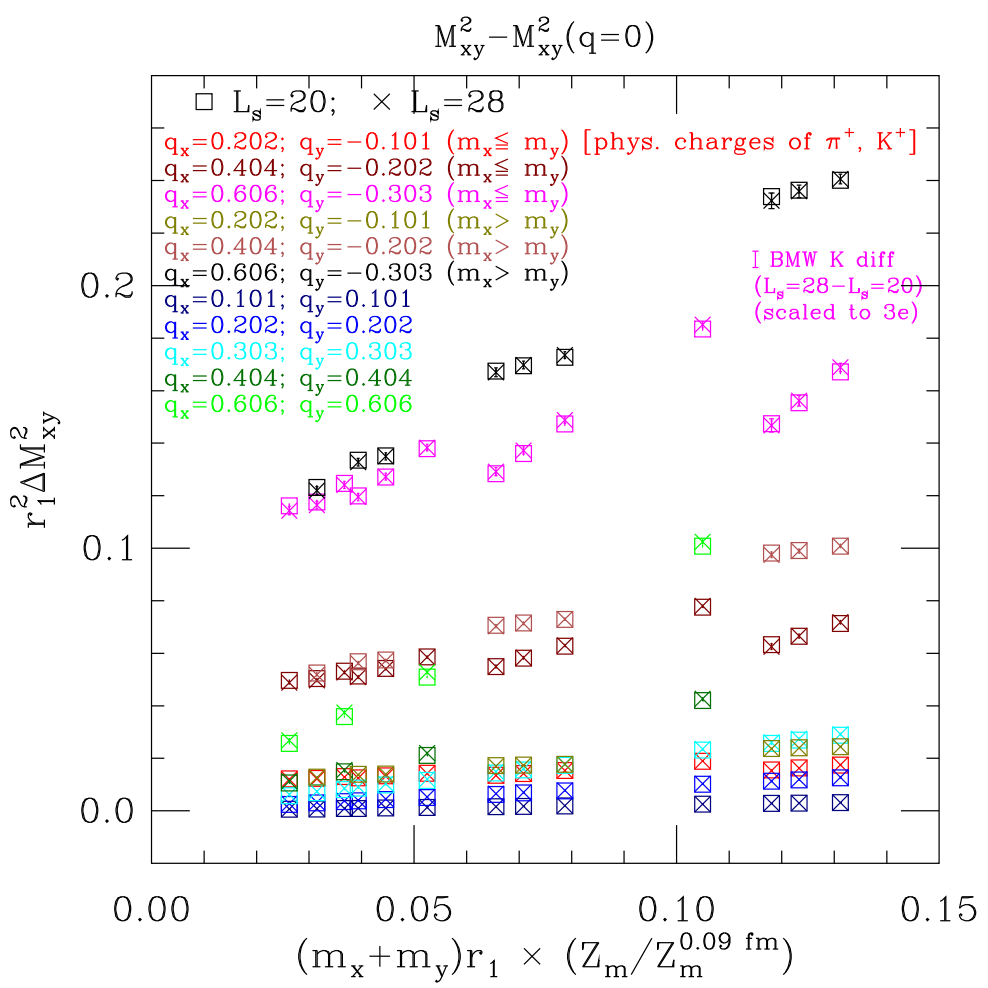

Figure 1: EM pseudoscalar splittings calculated on two coarse ensembles with $m_{l} / m_{s}=0.01 / 0.05$ and spatial sizes $L_{s}=20$ and $L_{s}=28 \mathrm{vs}$. the sum of the valence quark masses. The results for the different quark charge combinations are distinguished by color. The size of the finite volume effects found by the BMW group [7] is shown in magenta in the upper right and is scaled to charge $3 e$, to correspond to our magenta points plotted slightly below that. The finite volume effects we see (difference between crosses and squares) are smaller than those found by BMW. 


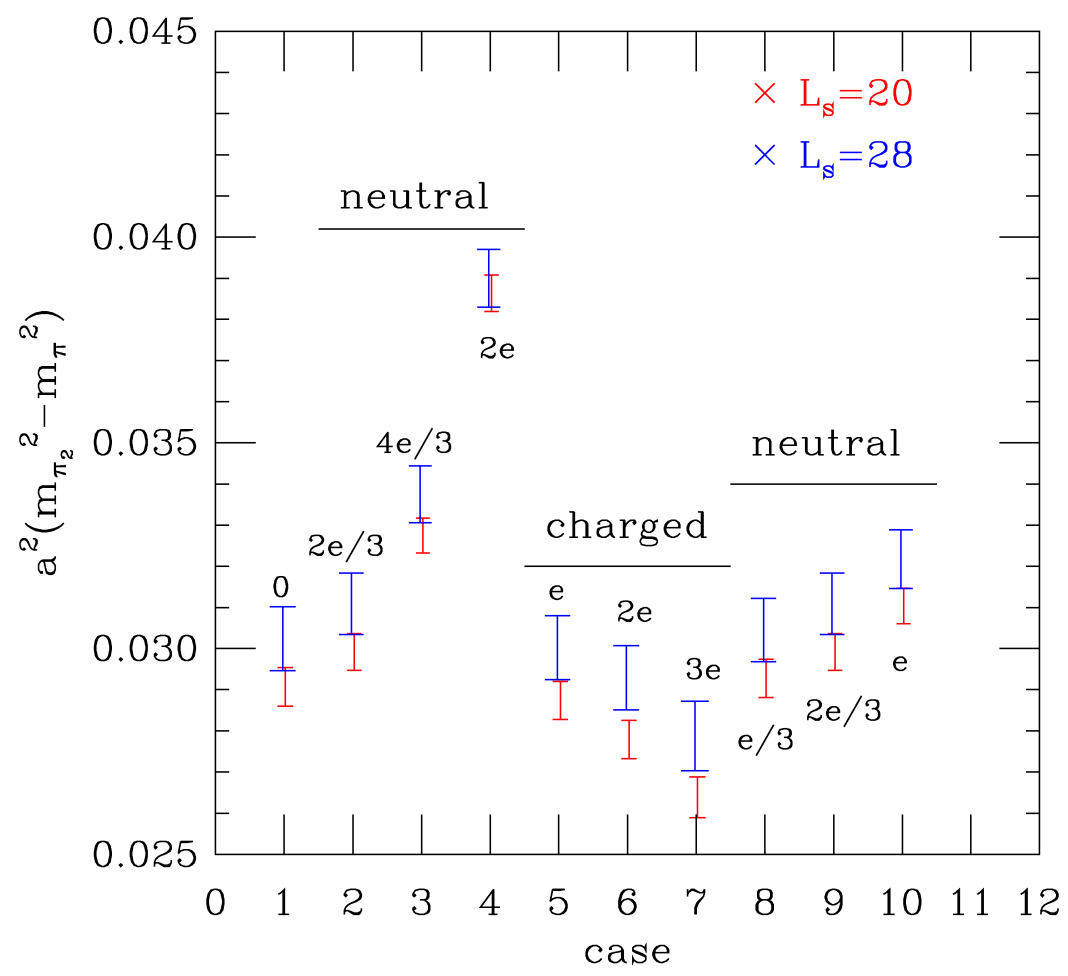

Figure 2: Taste splitting between the Goldstone pion and the second local pion on the coarse ensembles with $m_{l} / m_{s}=0.01 / 0.05$ and $L_{s}=20$ and $L_{s}=28$. The $x$-axis values are arbitrary labels for the mesons with various values of the quark charges. Case 1 is a pion made from uncharged quarks. The first group of neutral pions (cases 2-4) are essentially $\bar{u} u$ combinations, and the second neutral group (cases 8-10) are of the $\bar{d} d$ type (the $(2 e / 3,-2 e / 3)$ combination appears in both neutral groups). These groups are labeled by one of the quark charges (the other has an opposite sign). The middle group (cases 5-7), has quark charges with the physical ratio of (2:1); they are labeled by the total meson charge.

different $L_{s}$ in Table 2). This shows that, for charges larger than the physical charges, quark taste changing by high-momentum photons becomes a significant correction to that by high-momentum gluons. For charged pions, where annihilation into photons is forbidden, the effect is smaller (and negative), but still evident. The fit function Eq. (1.2) is based on the neglect of taste-violations caused by photons. For that reason, the analysis presented here focuses only on fits with physical quark charges. However, to the extent that photon-induced taste violations remain small compared with gluon-induced violations, which is arguably the case for all values of quark charges shown in Fig. \&, one should be able to expand the fit function in powers of $\alpha_{E M}$. This would allow such effects to be included by adding $\alpha_{E M}^{2}$ analytic terms to the fit function. We have made some preliminary fits along these lines to our full data set with quark charges up to three times the physical values, and they seem to work reasonably well.

Before leaving the subject of taste violations, we note that Fig. 2 2 shows that taste splitting is not very much affected by finite volume effects (compare the data in blue and red at $L_{s}=20$ and 28 for the above coarse ensembles).

We now fit, to Eq. (1.2) plus NNLO analytic terms, the EM splitting of the meson spectrum 
using the data with the physical electron charge $e$ (i.e., mesons made from combinations of $u$ and/or $d$ quarks, with $q_{u}=2 / 3 e$ and $q_{d}=-1 / 3 e$ ). Partially quenched points are included; in typical fits there are between 50 to 120 data points, with 20 to 30 fit parameters (depending on how many of the NNLO terms are included, and whether small variations with $a^{2}$ of the LO and NLO low energy constants are allowed). A typical fit is shown in Fig. 3, along with the unitary pions and kaons on each ensemble. The fit is uncorrelated: the covariance matrix is nearly singular, and the statistics are insufficient to determine it with enough precision to yield good correlated fits. Based on the fits, our preliminary determination of the correction to Dashen's theorem in the continuum limit and at physical quark mass is $\varepsilon=0.65(7)(14)$, where the errors are statistical and systematic, respectively. The systematic error comes largely from variations in $\varepsilon$ when the assumptions going into the chiral/continuum fit are changed. Systematic effects are still under investigation. In particular, based on the currently small finite volume effects seen in our data, we do not include a finite volume error here. However, we suspect that better statistics on our larger lattices will yield a statistically significant finite volume error. Our result is compatible with previous ones: 0.60(14) (statistics only) [10], 0.628(59) (statistics only) [9], and 0.70(4)(8) [7]. With our value for $\varepsilon$ from above, our preliminary estimate for the EM uncertainty in $m_{u} / m_{d}$ is reduced to 0.022 [11], which is about half of our previous error shown in Table 1 .

Acknowledgements: Computations for this work were carried out with resources provided by the USQCD Collaboration and the National Energy Research Scientific Computing Center, which are funded by the Office of Science of the U.S. Department of Energy; and with resources provided by the National Science Foundation, and the National Institute for Computational Science and the Texas Advanced Computing Center, which are funded through the National Science Foundation's Teragrid/XSEDE Program. This work was supported in part by the U.S. National Science Foundation under grants PHY0757333 (C.D.) and PHY0903571 (L.L.). Fermilab is operated by Fermi Research Alliance, LLC, under Contract No. DE-AC02-07CH11359 with the United States Department of Energy. For this work we employ QUDA [12].

\section{References}

[1] A. Bazavov et al., Rev. Mod. Phys. 82, 1349 (2010), [arXiv:0903.3598 [hep-lat]]; C. Aubin et al. [MILC Collaboration], Phys. Rev. D 70, 114501 (2004), [arXiv:hep-lat/0407028]; A. Bazavov et al. [The MILC Collaboration], PoS(LAT2009)079 (2009), [arXiv:0910.3618].

[2] S. Basak et al. [MILC Collaboration], PoS(LAT2008)127 (2008), [arXiv:0812.4486 [hep-lat]].

[3] C. Bernard and E. D. Freeland, PoS LATTICE 2010, 084 (2010), [arXiv:1011.3994 [hep-lat]].

[4] A. Torok et al., PoS LATTICE 2010, 127 (2010).

[5] R. Dashen, Phys. Rev. 183, 1245 (1969).

[6] G. Colangelo et al., Eur. Phys. J. C 71, 1695 (2011) [arXiv:1011.4408 [hep-lat]]

[7] A. Portelli et al., [arXiv:1201.2787 [hep-lat]].

[8] J. Bijnens and N. Danielsson, Phys. Rev. D 75, 014505 (2007) [hep-lat/0610127].

[9] T. Blum et al., Phys. Rev. D 82, 094508 (2010) [arXiv:1006.1311 [hep-lat]]. 


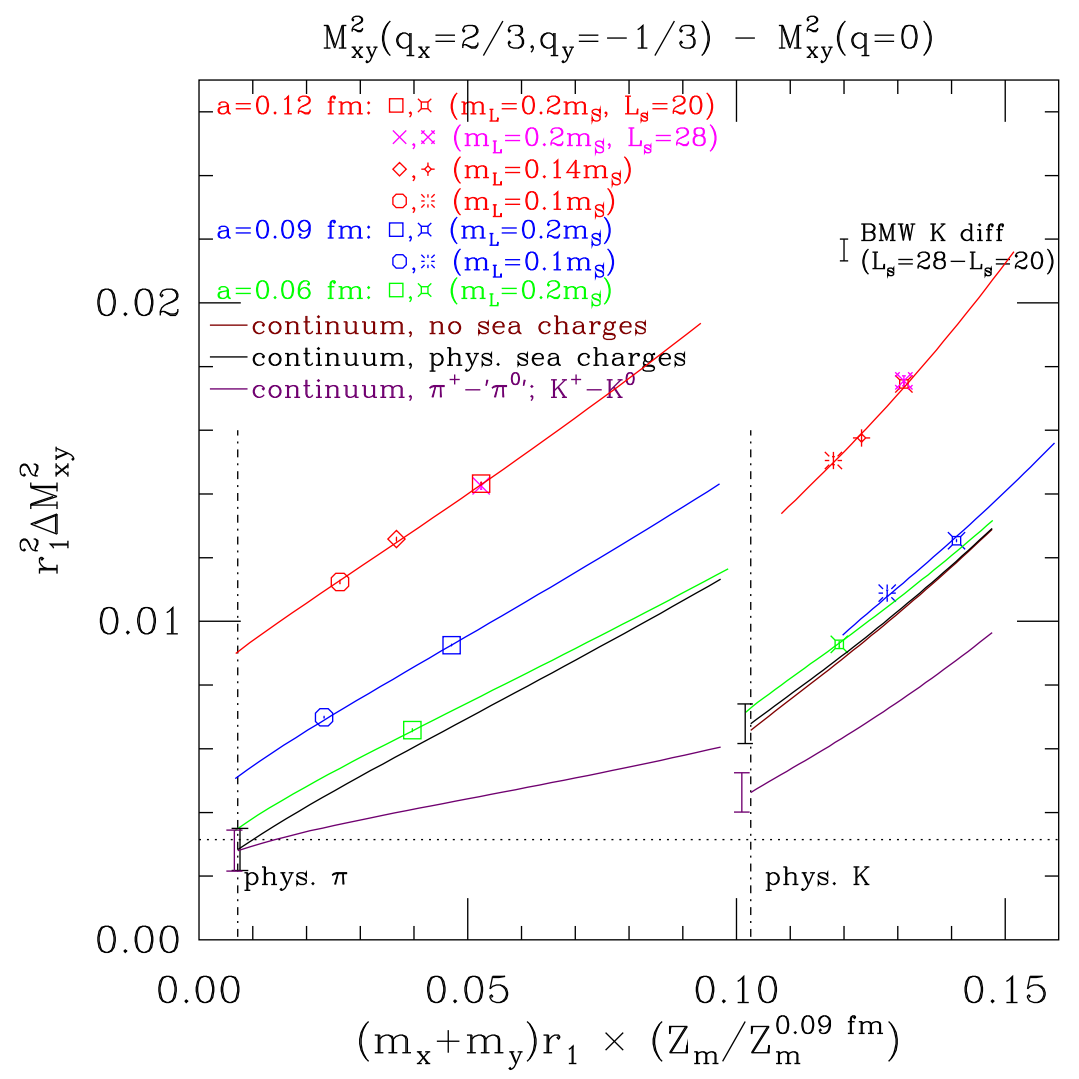

Figure 3: Our preliminary results for electromagnetic corrections in pseudoscalar mesons. Plotted is the difference in the squared mass of mesons with charged valence quarks and neutral valence quarks $v s$. the sum of the valence quark masses. Only a small subset of the data that was used in the fit is shown in the plot. This fit had 55 data points, 26 parameters, and an (uncorrelated) $p$ value of 0.09 . The red, blue and green curves correspond to three different lattice spacings and include the effects of taste breaking. The vertical dot-dashed lines correspond to the quark masses of the $\pi$ and $K$ mesons. The purple curves are the continuum limits for $K^{+}-K^{0}$, and for $\pi^{+}-^{\prime} \pi^{0 \prime}$ (left), where the ' $\pi^{0 \prime}$ is the average of a neutral pion with charges $(2 / 3 e,-2 / 3 e)$ and $(1 / 3 e,-1 / 3 e)$. The latter is an approximation to the physical $\pi^{0}$, which would also be affected by quark annihilation terms not included in our calculation. The horizontal dotted line is the physical value of the pion electromagnetic mass splitting and is in close agreement with our calculation. The result for the $K$ is larger than for the pion, giving a correction to the lowest order of Dashen's theorem by a factor of $\varepsilon=0.65(7)$. In the upper right we show the expected size of finite volume effects for the kaon from the BMW calculation [7]. It should be compared with the (tiny) difference between the red square and magenta cross, or 'fancy' red square and 'fancy' magenta cross.

[10] A. Portelli et al. [Budapest-Marseille-Wuppertal Collaboration], PoS LATTICE 2010, 121 (2010) [arXiv:1011.4189 [hep-lat]].

[11] D. Toussaint, PoS(Lattice 2012)159.

[12] M. A. Clark et al., Comput. Phys. Commun. 181, 1517 (2010) [arXiv:0911.3191 [hep-lat]]. R. Babich et al., International Conference for High Performance Computing, Networking, Storage and Analysis (SC), 2011 [arXiv:1109.2935 [hep-lat]]. 